\title{
Chronische Entzündung und Extrazelluläre Matrix ECM: Ausgangspunkt für ein anti-inflammatorisches Interventionsschema
}

\author{
Herbert Schwabl, Cécile Vennos \\ Padma AG, CH-Schwerzenbach
}

Di. ie von einer Entzündung im Organismus ausgelösten Vorgänge sind vielfältig und komplex: vom Auftreten der primären Entzündungszeichen (Rötung, Schwellung, Schmerz, Wärme, Funktionsverlust) bis zu Veränderungen des Elektrolythaushalts und verschiedenen immunologischen Vorgängen. Obwohl schmerzhaft, ist eine Entzündung normalerweise ein Heilungsprozess.

Bei einer Infektion oder einer Verletzung reagieren Immunzellen, indem sie mittels Mehrfachimpulsen andere Zellen rekrutieren und aktivieren. Solange mikrobielle und körpereigene pro-inflammatorische Stimuli vorherrschen, wird das entzündliche Geschehen über positive Rückkoppelungsmechanismen konsequent auf Höchsttouren gebracht. Kurz gefasst laufen bei einer Akutphasenreaktion folgende Prozesse ab [1]:

- Makrophagen locken über chemotaktische Faktoren neutrophile Granulozyten an.

- Makrophagen und Neutrophile schütten reactive oxygen intermediates (ROI), reactive nitrogen intermediates (RNI) und Hyaluronidase aus, welche Komponenten der extrazellulären Matrix (extracellular matrix ECM) zersetzen.

- Die Hyaluron-Fragmente wirken ihrerseits als Verletzungssignale und induzieren die Freisetzung von weiteren chemotaktischen Faktoren und Matrix-Metalloproteinasen (MMPs) durch Makrophagen, die die ECM weiter abbauen.

- Tumornekrose-Faktor (TNF)- $\alpha$ aus Makrophagen aktiviert Neutrophile, die durch Ausschüttung von Elastase
Hintergrund: Entzündungsvorgänge induzieren eine inflammatorische Signalkaskade, die sich über positive Feedbackmechanismen am Laufen hält. Können diese Rückkopplungen trotz Beseitigung der eigentlichen Entzündungsursache nicht durchbrochen werden, wird die Entzündung chronisch. Chronische Entzündungen sind nicht mehr Teil des natürlichen Heilungsprozesses, sondern schädigen als eigener Aspekt der Pathogenese das Gewebe oft mehr als die ursprüngliche Entzündungsursache. Das komplexe Entzündungsgeschehen ist mehr als das Zusammenspiel der zellulären Protagonisten und ihrer Zytokine. Die Komplementärmedizin bietet mit dem System der Grundregulation einen erweiterten funktionalen Raum, in dem Immun- und Mesenchymzellen mit der extrazellulären Matrix ECM, dem strukturierten Interstitium sowie der Blutendstrombahn, Nervenendigungen und Lymphbahnen interagieren. Zielsetzung und Methode: Die Struktur der Entzündungsreaktion aus immunologischer Sicht wird dem System der Grundregulation gemäss A. Pischinger und $\mathrm{H}$. Heine gegenübergestellt. Es wird analysiert, ob sich daraus neue integrative Ansätze für die antientzündliche Therapie sowie die Prävention und Nachsorge von chronisch-entzündlichen Erkrankungen ergeben. Ergebnisse: Zur Beendigung von Entzündungsreaktionen sind nicht nur verschiedene anti-inflammatorische Moleküle nötig; insbesondere bei chronischen Entzündungen ist die Wiederherstellung der Regelfähigkeit des Grundsystems unerlässlich. Erst das Triggern und Verstärken von anti-inflammatorischen Impulsen leitet die eigentliche Heilungsphase ein. Demnach lässt sich ein dreistufiges, auf die ECM fokussiertes Interventionsschema ableiten: Durch die drei Therapiephasen "Reload-Protect-Trigger" können die verschiedenen Funktionen der ECM angesprochen werden. Dieses integrative Konzept wird an Beispielen erklärt und in ein praxistaugliches Schema übergeführt. Dies erlaubt es, im Alltag auf einfache Weise den Bezug individueller Therapieansätze zum dreistufigen Funktionsmodell der ECM zu beurteilen. Die integrative Sichtweise erscheint insofern notwendig, als gerade im Bereich der chronischen Entzündungen viele Massnahmen eingesetzt werden, eine übergeordnete Leitlinie jedoch oft fehlt.

Schlüsselwörter: Extrazelluläre Matrix ECM, Grundsystem, chronische Entzündung, Phytotherapie, Komplementärmedizin

\section{Chronic Inflammation and Extracellular Matrix ECM:}

\section{Starting Point for an Anti-Inflammatory Intervention Scheme}

Background: In inflammation, a signal cascade is set in motion which sustains itself via positive feedback mechanisms. If, in spite of the elimination of the underlying cause of the inflammation, these feedback loops cannot be interrupted, the inflammation becomes chronic. Chronic inflammations stop being a part of the normal healing process. As a discrete aspect of pathogenesis they often harm the body tissue more than the initial cause of inflammation. The complex inflammatory processes encompass more than the interaction of cellular protagonists and their cytokines. Using the principle of the 'ground regulation' complementary medicine offers an expanded functional space, in which immune cells and mesenchymal cells interact with the extracellular matrix ECM, the structured interstitium, as well as the capillary blood system, nerve endings and lymph vessels. Objective and method: The immunologic view of the inflammatory reaction is compared to the system of ground regulation according to A. Pischinger and $\mathrm{H}$. Heine. The question is asked whether, from this comparison, new integrative approaches to anti-inflammatory therapy result, as well as to prevention and aftertreatment of chronic inflammatory diseases. Results: Not only are different anti-inflammatory molecules necessary for termination of inflammatory reactions, especially in chronic inflammations, the regeneration of the regulatory capacity of the system of ground regulation is essential. Only triggering and amplifying anti-inflammatory impulses initiates the actual healing phase. From this a three-step intervention scheme can be derived which is focused on the ECM: through the three phases of therapy, "reload-protect-trigger", different functions of the ECM can be addressed. By means of examples this integrative concept is explained and transferred into a scheme applicable in practice. This facilitates assessment of the relevance of individual treatments to the three-step functional model of the ECM in everyday life. The integrative perspective seems necessary, especially in the area of chronic inflammations where a multitude of methods are applied but an overall therapy concept is often missing.

Key words: extracellular matrix ECM, ground regulation, chronic inflammation, herbal medicine, complementary medicine, CAM 
zur Zersetzung der ECM beitragen und wiederum die MMPs aktivieren. Die Kettenreaktion verstärkt sich.

- MMPs aktivieren transforming growth factor (TGF)- $\beta$ aus Makrophagen, den stärksten chemotaktischen Faktor. Elastase baut zusätzlich das TGF- $\beta$ Bindungsprotein $a b$ und unterstützt so die Aktivierung von TGF- $\beta$.

Normalerweise wird die akute Entzündungsantwort durch sich gegenseitig regulierende Mechanismen lokal begrenzt und so die Verbreitung der Entzündungsmoleküle in die Blutbahn verhindert. Bei diesem endogenen antiinflammatorischen Signalweg wird eine Reihe von löslichen entzündungshemmenden Mediatoren aktiv [2]. Dazu gehören anti-inflammatorische Zytokine und Hormone. Die Zytokine Interleukin (IL)-10, TNF-binding Protein und TGF- $\beta$ unterbinden spezifisch die Freisetzung von TNF- $\alpha$ und anderen proinflammatorischen Molekülen. Hormone wie adrenale Glukocorticoide, Adrenalin, Noradrenalin und $\alpha$-melanozytenstimulierendes Hormon hemmen die Zytokinsynthese und die intrazelluläre Signaltransduktion. Zusätzlich akkumulieren lokale Effektoren, z.B. Prostaglandin E2, Heat-shock protein und Spermin an Orten von Gewebeverletzungen und Infektionen und inhibieren die Makrophagenaktivierung und damit die Zytokinsynthese.

Die vom Immunsystem angestrebte Zerstörung von Verletzungsbereichen und infizierten Zellen sowie der Abbau von Debris und die Reparatur des Gewebes müssen genau aufeinander abgestimmt sein. Ist in dieser Kaskade zu einem Zeitpunkt ein Schritt blockiert, wird der vorangehende Prozess in eine Warteschleife umgeleitet und es kann z.B. zum Stau von Lymphozyten-Aggregaten in der ECM kommen, oder eine überschiessende Produktion von ECM-Komponenten, als Reparaturantwort der Fibroblasten, führt zu Narbenbildung [2].

In den meisten Fällen klingt eine Entzündung von selbst wieder ab und es kommt zur Heilung, der Normalzustand ist wieder hergestellt. Chronische Entzündungen sind jedoch pathogene Prozesse, die systematisch fortschreiten und den Organismus schädi- gen; die Entzündung ist ausser Kontrolle geraten. Oft entwickelt sich ein Krankheitsbild, bei dem nicht mehr zwischen der Schädigung durch die ursprüngliche Ursache und der Schädigung durch die Entzündung unterschieden werden kann.

\section{Chronische Entzündung als ungelöstes Problem}

Die Verwendung des Wortes „ungelöst“ in Zusammenhang mit chronischinflammatorischen Prozessen ist durchaus in einem doppelten Wortsinn zu verstehen. Zum einen kann die Kaskade der sich aufschaukelnden Entzündungsabläufe nicht gestoppt werden und die Steuerungsmechanismen des Organismus können diesen Programmfehler alleine nicht mehr beheben. Zum anderen steht therapeutisch wenig zur Verfügung, um in diesen Prozess sanft aber dennoch zielgerichtet einzugreifen. Aus diesen Gründen ist die Prävention von chronischen Entzündungszuständen bzw. die Nachsorge nach chronisch-entzündlichen Erkrankungen besonders wichtig.

Bei chronischen Erkrankungen steht klar die kausale Abklärung im Vordergrund. Findet man jedoch die pathogene Ursache nicht, empfiehlt sich die systemische Kontrolle der Entzündungsprozesse. Obwohl für einige Krankheiten mit vermutetem entzündlichem Ursprung eine Infektion als Ursache gefunden wurde (z.B. Helicobacter pylori-abhängige, chronisch ulzerierende Gastritis [3]), richten in diesem und vielen anderen Fällen die inflammatorischen Prozesse weit grösseren Schaden an als die Mikroorganismen selbst. Zwar geht die Suche nach Infektionen als mögliche Ursache bei Krankheiten wie Multiple Sklerose, Rheuma und Arteriosklerose weiter [4, 5], die Behandlung der Entzündung an sich bleibt in diesen Fällen aber immer noch eines der primären therapeutischen Ziele.

Je nachdem, welcher Schädigungsmechanismus im Vordergrund steht, sind therapeutische Massnahmen für das akute und das chronische Stadium, aber auch für die postinfektiöse Phase, differenziert zu beurteilen und auszuwählen. Komplementärmedizinische Verfahren zeigen ihre Stärke besonders in der Prävention, im chronischen Verlauf, bei der Unterstützung der Ausheilung und in der Nachsorge. Chronischen Entzündungen liegt ein multikausales Geschehen zugrunde, welches ein pragmatisches, ganzheitlich orientiertes Vorgehen rechtfertigt [6].

\section{Die Bedeutung des Systems der Grundregulation}

Um das chronisch-entzündliche Geschehen besser zu verstehen, ist eine Aufweitung des konzeptiven Rahmens notwendig. Dabei wird schnell deutlich, dass eine rein zelluläre Betrachtung den komplexen Abläufen nicht gerecht wird [7]. Neuere biomedizinische Ergebnisse zeigen, dass eine solche Erweiterung des Blickwinkels auch bei der Tumorgenese nötig ist [8].

Die ganzheitsmedizinische Forschung bietet hier eine Sichtweise, die das zelluläre Geschehen und das umgebende Milieu als eine zusammenhängende organisatorische Einheit beschreibt: das System der Grundregulation, als eine integrale Funktionseinheit der Gefässendstrombahn, der Bindegewebszellen und der vegetativ-nervalen Endformation [9, 10]. Die Körperzellen stehen mit ihrer Umgebung, dem Extrazellulärraum, in einer untrennbaren, wechselseitigen Beziehung, wobei die ECM als ein jeder Zelle vorgeschaltetes Molekularsieb wirkt. Konkret besteht das System der Grundregulation aus folgenden Komponenten:

1. Abkömmlinge der embryonalen Mesenchymzellen (u.a. Fibroblasten), die die Moleküle der ECM bilden, sowie die ECM-abbauenden Makrophagen und Mastzellen.

2. Der ECM, d.h. der strukturierten Zwischenzellsubstanz, bestehend aus Proteoglykanen (PG) und Glucosaminoglykanen (GAG), Strukturglykoproteinen (Kollagen, Elastin) und Vernetzungsglykoproteinen (u.a. Fibronektin, Laminin). Die ECM ist ein durchgehender Funktionsraum, der die Organe und somit den gesamten Körper durchzieht.

3. Der Endstrombahn des Gefässsystems, d.h. des Kapillarnetzes. Da- 


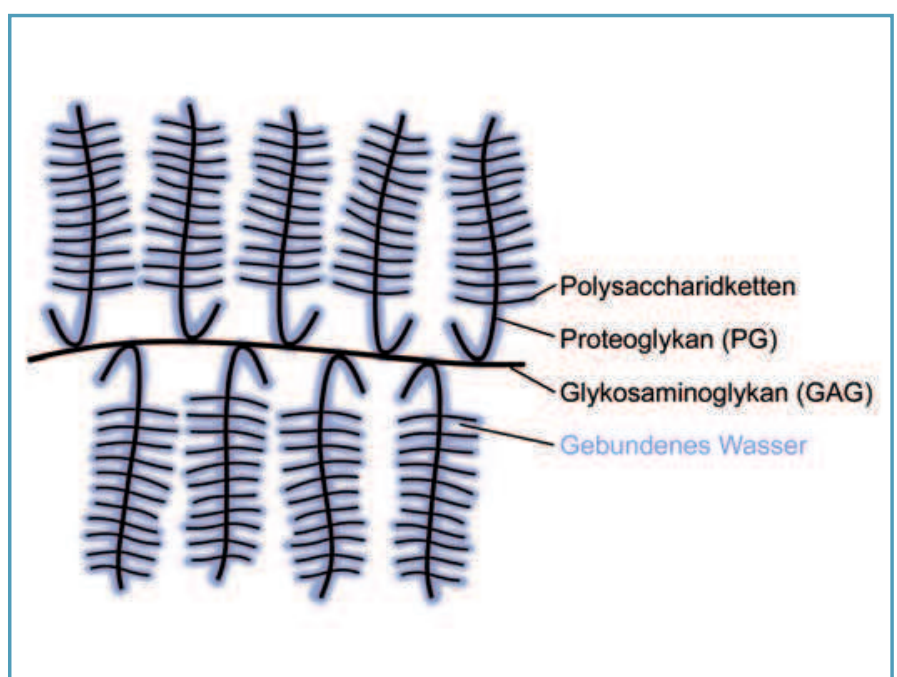

Abb. 1. Schema der Matrix-Struktur. Das Gerüst der extrazellulären Matrix (ECM) besteht aus einem Netzwerk von Glykosaminoglykanen (GAG), darauf aufgesetzt die Proteoglykane (PG) und die kammartigen Polysaccharidketten. Durch die sich abstossenden negativen Ladungen ist es straff gespannt. Die Schicht gebundenen Wassers ist schematisch angedeutet (nach [11]).

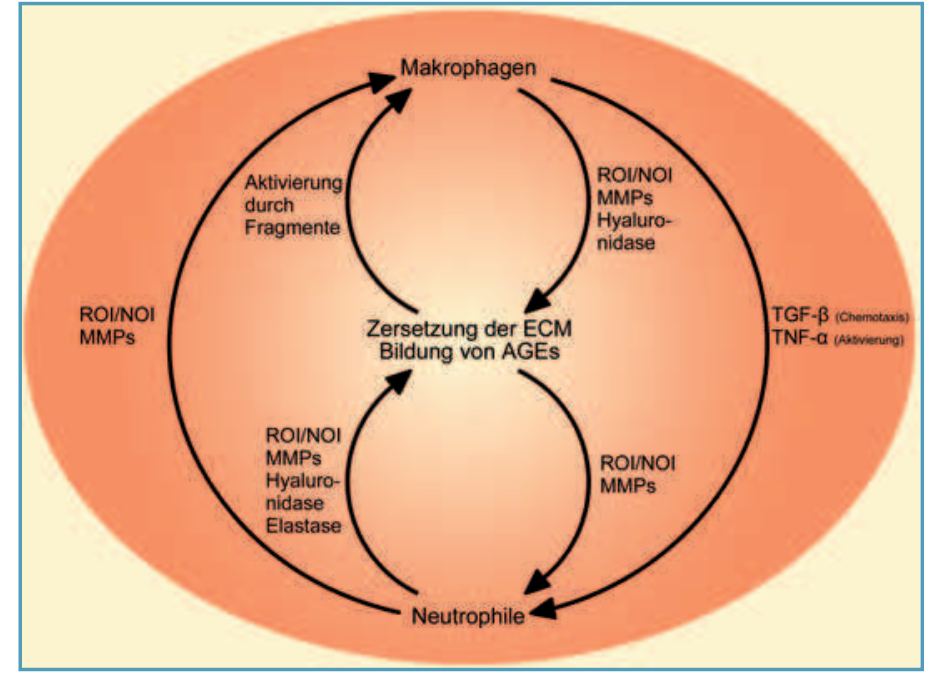

Abb. 2. Die extrazelluläre Matrix im Zentrum der Entzündung. Störungen in der ECM und Abbauprodukte ihrer Komponenten interagieren mit Immunzellen und der inflammatorische Prozess wird über positive Feedbackmechanismen am Laufen gehalten. (AGEs: advanced glycation endproducts; ECM: extrazelluläre Matrix; MMPs: Matrix-Metalloproteinasen; $\mathrm{ROI} / \mathrm{NOI}$ : reactive oxygen/nitrogen intermediates; TGF- $\beta$ : transforming growth factor beta; TNF- $\alpha$ : Tumornekrose-Faktor alpha.) durch besteht unter anderem eine Ankopplung an die Hormone des Endokrinums.

4. Nervenenden und damit einer Rückkopplung an das zentrale Nervensystem. Über die ECM bildet das System der Grundregulation eine Verschaltung zwischen dem Nervenund dem endokrinen System.

5. Lymphbahnen. Die offenen Lymphgefässe drainieren die ECM von zellulären Stoffwechselendprodukten.

Die ECM wirkt also als Mittlerin zwischen den Transportsystemen (Blutund Lymphgefässen), den Nervenenden und den Körperzellen. Die PG/ GAGs bilden das strukturelle Grundelement der ECM (Abb. 1). Aufgrund des siebartigen Aufbaus und der negativen Ladung des Proteinnetzwerkes ist ein Grossteil des Zellwassers nahe an den makroskopischen, strukturierten Oberflächen angelagert. Im Abstand von etwa $2-3 \mathrm{~nm}$ (etwa 10 Molekülschichten) um die Grundelemente hat dieses „gebundene Wasser“ andere Eigenschaften als ungebundenes, freies Wasser. Es steht nicht als Lösungsraum für Moleküle zur Verfügung. Die PG/GAGs strukturieren damit ein poröses Polysaccharidgel mit enormer Oberfläche. Die in der Grundsubstanz ent- haltenen Elektrolyte und der daraus resultierende $\mathrm{pH}-$ Wert spielen zudem eine entscheidende Rolle für die Beschaffenheit der Grundsubstanz (Solund Gelzustand) und damit für die Permeabilität. Lösliche Stoffe diffundieren dagegen nur in der freien Wasserphase und werden durch sie wie durch ein Kanalsystem transportiert.

Die kammartige Struktur, damit verbunden die innere Spannung des PG/ GAG-Netzwerkes im Sol-/Gelzustand, ist nur durch den negativen Ladungszustand möglich. Insbesondere die Sulfatgruppen der PG (Chondroitin-, Dermatan-, Heparan- und Keratansulfat) sind notwendig, um den optimalen Ladungszustand aufrechtzuerhalten. Wichtig sind auch Spurenelemente (Kieselsäure $\mathrm{SiO}_{2}$, Vitamine und sekundäre Pflanzenstoffe wie z.B. Polyphenole oder Carotinoide), um das negative Redoxpotential zu stärken.

Die ECM ist in ihrem Normalzustand also in einem labilen Gleichgewicht. Analog dazu basiert der entzündungsfreie Zustand nicht passiv auf dem Nichtvorhandensein entzündlicher Stimuli. Für seinen Erhalt sind spezifische positive Aktivitäten notwendig, um Reaktionen auf unterschwellige Entzündungsreize zu unterdrücken [12].

In der ECM ansässige Immunzellen bauen ständig deren Bestandteile ab und sind in latenter Bereitschaft, auf inflammatorische Trigger mit einer Entzündung zu reagieren. Gleichzeitig sind die Fibrozyten konstant mit dem Aufbau von ECM-Komponenten beschäftigt [10]. Dies schafft die Voraussetzung für Veränderungen durch permanenten Auf- und Abbau, zugleich bleibt das Gesamtsystem in steter Alarmbereitschaft. Diese physiologisch wichtige Eigenschaft der Plastizität kann nur unter den optimalen Ladungsbedingungen aufrechterhalten werden.

\section{Die ECM während einer Entzündung}

Wie eingangs beschrieben, wird eine Entzündung von matrix-ständigen Makrophagen initiiert, wobei auch das Komplementsystem Startsignale setzen kann. Mastzellen werden durch IgEAntikörper aktiviert. Die freigesetzten Entzündungsmediatoren sorgen für die erhöhte Permeabilität der ECM und locken durch chemotaktische Faktoren Neutrophile an, die aus den Kapillaren in die ECM einwandern. Makrophagen und Neutrophile schütten ROI, RNI und Hyaluronidase aus [1] (Abb. 2).

Oxidativer Stress führt bei glykosi- 
lierten Proteinen, welche eine entscheidende Rolle für die Struktur der ECM spielen, zur Bildung von advanced glycation endproducts (AGE). Durch ROI werden die AGE unter Einschluss von Lipiden zu grossen unlöslichen Molekülen polymerisiert. Dieser Vorgang kann als „Verschlackung“ der ECM bezeichnet werden [13] und führt zu einer positiven Rückkopplung, die im chronisch entzündlichen Verlauf einen Rückstau erzeugt: ROI erzeugen AGE, die wiederum oxidativen Stress induzieren [14].

Die von aktivierten Makrophagen und Neutrophilen ausgeschüttete Hyaluronidase und proteolytische Enzyme spalten die PG/GAGs. Die dadurch entstehenden Hyaluron-Fragmente wirken ihrerseits als Verletzungssignale und induzieren via Makrophagen die weitere Freisetzung von chemotaktischen Faktoren und Proteasen, welche Kollagen, PG und Fibronektin abbauen.

TNF- $\alpha$ aus Makrophagen aktiviert Neutrophile, die nun vermehrt Elastase frei setzen. Die Kettenreaktion verstärkt sich, indem Elastase und ROI wiederum die Proteasen aktivieren.

Proteasen aktivieren Makrophagen zur Produktion von TGF- $\beta$, dem stärksten chemotaktischen Faktor. Elastase baut zusätzlich das TGF- $\beta$-Bindungsprotein $a b$ und unterstützt so die Aktivierung des anti-inflammatorisch wirksamen TGF- $\beta$.

Im chronischen Entzündungsverlauf spielen die Zerstörung der Struktur der ECM und die daraus entstehenden Zersetzungsprodukte eine wichtige Rolle. Deshalb ist es wichtig, die ECM durch Strukturkomponenten zu schützen und der ständigen Produktion von Zell- und Matrixbruchstücken entgegenzuwirken. Zusätzlich muss die Bildung von AGE verhindert werden. Diese Bedingungen können einerseits durch Ausleitung und Sanierung von Störquellen, andererseits durch eine generelle Minderung des Risikoprofils, insbesondere der oxidativen Stressbedingungen, herbeigeführt werden.

\section{Wie wird eine Entzündung wieder beendet?}

Die Entscheidung, eine Entzündung wieder zu beenden, wird im Entzündungsverlauf relativ spät getroffen. Die antibakteriellen und Gewebe abbauenden Aktivitäten des Immunsystems und die inflammatorischen Prozesse müssen derart modifiziert werden, dass Gewebereparatur und die epitheliale Heilung gefördert werden. So müssen zum Beispiel die Freisetzung von Elastase aus Granulozyten unterdrückt, schon freigesetzte Elastase gehemmt und die Elastase-Restvorräte abgebaut werden. Das Timing ist dabei entscheidend - die Immunabwehrmechanismen dürfen nicht heruntergefahren werden, solange eine Wunde noch offen für Erreger ist.

Sobald jedoch von Gewebezerstörung auf Gewebeheilung umgeschaltet wird, wird das Zell- und Zytokinensemble neu gruppiert. Dabei können bestimmte Zytokine pro- oder anti-inflammatorisch wirken, je nach Zeitpunkt und Kontext.

Die Dramaturgie der Gewebeheilung respektive des antientzündlichen Geschehens wird damit eingeleitet, dass Makrophagen abgetötete Bakterien und Bruchstücke geschädigter Körperzellen aufzunehmen und zu verdauen beginnen [1]. Dabei sezernieren sie Proteaseinhibitoren, u.a. secretory leukocyte protease inhibitor (SLPI), welche anti-inflammatorisch und wundheilend wirken. SLPI unterdrückt die Freisetzung von Elastase und ROI durch TNF- $\alpha$-stimulierte Neutrophile und hemmt die bereits freigesetzte Elastase. Gleichzeitig schützt es TGF- $\beta$ und deaktiviert durch synergistische Wirkung die Neutrophilen. CD44-positive Makrophagen bauen die Hyaluronfragmente ab. Durch die Eliminierung dieses chemotaktischen Verletzungssignals werden keine frischen Neutrophilen rekrutiert und die bereits anwesenden leiten ihrerseits die Apoptose ein. Dieser physiologische Abbau von Leukozyten hilft ebenfalls, die Homöostase im Gewebe und im Blutserum wiederherzustellen [9]. Die Makrophagen werden durch die Phagozytose von untergegangenen Neutrophilen zur Ausschüttung von entzündungshemmendem TGF- $\beta$ stimuliert, das die Gewebeheilung fördert. TNF- $\alpha$ regt die Makrophagen an, IL-12 freizusetzen. Dieses induziert wieder- um in den Lymphozyten die Interferon (IFN)- $\gamma$-Abgabe, welches die Chemokinproduktion unterdrückt.

Die Notwendigkeit jedes dieser Schritte könnte fälschlicherweise zum Gedanken verleiten, dass ein Entzündungsprozess relativ einfach zu unterbrechen sei. Dem ist jedoch nicht so, unter anderem deshalb, weil viele Signale redundant sind. Eine Unterbrechung der Entzündung muss daher durch die parallele Hemmung verschiedener Signalwege auf mehreren Ebenen gleichzeitig erfolgen. Zusätzlich ist das Einsetzen von bekannten Stopp-Signalen sehr kompliziert. Dazu kommt das therapeutische Dilemma, dass, je drastischer ein Agens eine Entzündung unterdrückt, desto grösser das Risiko der Verschlimmerung einer Infektion wird (vgl. Corticosteroide).

Die Beendigung einer Entzündung ist also ebenso wie die Ankurbelung der Entzündungsprozesse ein komplexes Zusammenspiel von zellulären und Matrixkomponenten. Die Feedbackmechanismen bei beiden Vorgängen und die Tatsache, dass dieselben Mediatoren einmal pro- und in einem anderen Kontext anti-inflammatorisch wirken können, macht das therapeutische Eingreifen schwierig.

Es gilt also, nach Auswegen zu suchen und eine Unterbrechung der Entzündungsreaktion an vielen Stellen $\mathrm{zu}$ triggern, so dass einerseits die Akutreaktion nicht vorzeitig abgebrochen wird, andererseits aber beginnende Stopp-Signale verstärkt werden und die Ausheilungsphase beginnen kann. Insbesondere ist es essentiell, im Entzündungsverlauf wichtige Steuerimpulse zu geben. Dies ist die eigentliche Domäne der so genannten Regulationstherapien.

\section{Ein Matrix-basiertes Interventionsschema}

In seinem Standardwerk klassiert $\mathrm{H}$. HEINE die therapeutischen Verfahren im Hinblick auf das System der Grundregulation in folgende vier Gruppen: Klassische Regulationstherapien (darunter Phytotherapie, Homöopathie, Chinesische und Indische Traditionelle Medizin, Physiotherapie, Balneologie), 


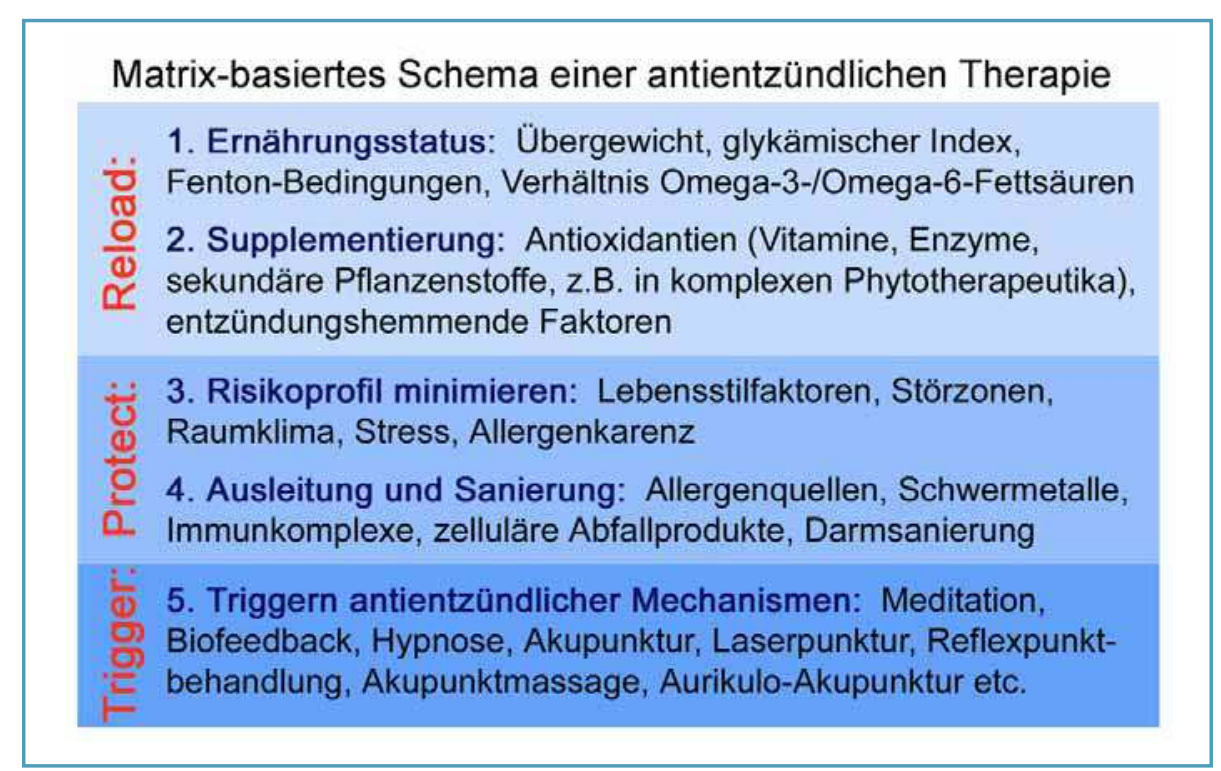

Abb. 3. Ein dreistufiges, Matrix-basiertes Schema einer antientzündlichen Therapie, das verschiedene Behandlungsansätze in ein Therapiekonzept integriert.

Therapieweisen mit gezielter Wirkung auf die Grundregulation (Akupunktur, Neuraltherapie, Bioresonanz), Therapieweisen mit systemischer (unspezifischer) Anregung der Grundregulation (Kneipp-Verfahren, Diätetik, psychischmentale Verfahren) und Ab- und Ausleitende Verfahren (Schröpfen, Purgieren, etc.) [10].

Als fundierter Praktiker weist 0 . Bergsmann im Grundlagenwerk zum System der Grundregulation einen funktionalen Weg: Beseitigung regulationsstörender Faktoren - Ausgleich von Defiziten - Abbau von Feedbackmechanismen [9].

Im Hinblick auf den bereits dargestellten Ablauf des chronisch-entzündlichen Geschehens erscheint es uns sinnvoll, den von Bergsmann vorgegeben Weg zu einem funktional auf die ECM ausgerichteten Therapieschema in drei Stufen auszubauen (Abb. 3):

- Reload: die ECM aufladen durch Diätetik und Zufuhr von essentiellen und anti-inflammatorischen Substanzen.

- Protect: das Risikoprofil durch Lebensstilmassnahmen verbessern, exogene Störfaktoren mindern. Ausleitung und Sanierung von Problemzonen in der ECM.

- Trigger: Setzen von Mehrfachimpulsen, um den Prozess in die antientzündliche Phase überzuführen.
Erste Voraussetzung einer solchen Therapieabfolge ist die Wiederherstellung der Regelfähigkeit des Organismus und die Regenerierung der extrazellulären Matrix (Stufe Reload). Dabei kommt u.a. dem Ernährungsstatus und der Supplementierung eine hohe Bedeutung zu, wobei Stoffe mit antioxidativem und entzündungshemmendem Potential, z.B. Vitamine oder sekundäre Pflanzenstoffe, eingesetzt werden können.

In einem zweiten Schritt wird eine Verbesserung des allgemeinen Entzündungsstatus angestrebt, so dass der Organismus optimal auf proinflammatorische Reize reagieren kann. Chronische entzündliche Stimuli werden vermindert und das Immunsystem so vor einer schleichenden Erschöpfung geschützt (Stufe Protect). Hier gilt es besonders, Risikofaktoren zu vermeiden und durch Ausleitungstherapien oder Sanierung proinflammatorische Reizquellen zu beseitigen. Eine dauerhafte Umstellung der Lebensumstände und des Verhaltens hin zu einem ,antientzündlichen Lebensstil“ sind dabei entscheidend.

Tritt nun eine Entzündung ein, ist der Organismus viel besser in der Lage, adäquat zu reagieren. Bei der therapeutischen Unterstützung des Körpers während der Entzündung und zur Einleitung des Heilungsprozesses ist es wichtig, die Komplexität der inflammatorischen Kaskade zu berücksichtigen. Da viele Signale redundant sind, müssen die Prozesse an vielen Stellen gleichzeitig moduliert werden, ohne jedoch die Akutreaktion des Körpers vorzeitig zu blockieren. Diese Modulierung kann durch Triggern von körpereigenen antientzündlichen Mechanismen bzw. durch das Verstärken von beginnenden Stopp-Signalen geschehen (Stufe Trigger). Verschiedene Komplementärmedizinische Disziplinen wie z.B. Akupunktur, Reflexpunktbehandlung oder Biofeedback kommen hier in Frage.

\section{Beispiele für eine ECM-basierte Therapie}

An Beispielen soll nun die Bewertung von antientzündlichen Therapien gezeigt werden. Es ist zu beachten, dass eine Methode - je nach Spezifität - in verschiedenen Stufen des Therapieschemas eingesetzt werden kann. Natürlich sind weitere Beispiele denkbar und sinnvoll.

\section{Phytotherapie: \\ Pflanzliche Vielstoffgemische am Beispiel von Padma 28}

Ein Baustein eines solchen antientzündlichen Therapiekonzeptes können pflanzliche Vielstoffgemische, Einzelpflanzen, aber insbesondere auch abgestimmte Multipflanzen-Mischungen darstellen. Paradigmatisch und beispielhaft ist hier Padma 28 (Swissmedic Nr. 41125), eine Vielstoffrezeptur aus der Tibetischen Medizin, zu sehen. Studienresultate bestätigen den Einsatz des Vielstoffpräparates im vorgestellten Therapieprotokoll. Ähnliche Mechanismen und Gesichtspunkte könnten auch für andere Vielstoffgemische Gültigkeit haben:

\section{Stufe Reload}

Die vielfältigen sekundären Pflanzeninhaltsstoffe können als Antioxidantien und antientzündliche Faktoren in der Supplementierung angewendet werden [15-17].

\section{Stufe Protect}

In Situationen, die zu erhöhtem oxidativem Stress im Körper führen, und bei 
erhöhten Risikofaktoren dienen sie dem Schutz des Immunsystems. Durch seine durchblutungsfördernde Wirkung [18] kann Padma 28 auch die im Rahmen des antientzündlichen Therapieschemas durchgeführten Ausleitungstherapien unterstützen. Dabei weist die Pflanzenformel neben antioxidativen und anti-inflammatorischen Eigenschaften [15, 19] auch antibakterielles Potential [20] und eine antiproliferative Wirkung auf glatte Muskelzellen auf [12]. In-vitro-Versuche zeigten eine Hemmung von Matrix-Metalloproteinasen durch das Präparat, was für den Schutz der ECM-Strukturmoleküle von Bedeutung ist [21, 22].

\section{Akupunktur und Triggerpunkt- behandlung}

Eine Entzündung im peripheren Gewebe hat über das System der Grundregulation auch Einfluss auf die neuronale Signalweiterleitung im Hypothalamus. Neuronen des Zentralnervensystems können zum Beispiel Zytokine synthetisieren und exprimieren, welche dann wiederum eine Rolle in der neuronalen Kommunikation spielen [23, 24]. Diese Kommunikation ist bidirektional, da Zytokine die hypothalamisch-hypophysäre Freisetzung von Glukocorticoiden aktivieren und diese wiederum eine weitere Zytokinsynthese unterdrücken [25]. Zusätzlich können Zellen des Immunsystems Neuropeptide (inklusive Endorphine) produzieren, sowie Acetylcholin und andere Neurotransmitter.

\section{Stufe Protect}

Obwohl die Literatur nicht konsistente Resultate liefert, scheint Akupunktur beim Ernährungsstatus und bei Lebensstilfaktoren eine positive Wirkung $\mathrm{zu}$ haben. So zeigen einige Studien, dass Akupunktur zur Reduktion von Übergewicht beitragen kann, möglicherweise durch eine Erhöhung des Leptin- und des Endorphinspiegels [26, 27]. Ausserdem wirkt Akupunktur unterstützend bei der Raucherentwöhnung [28] und kann Stress oder dessen Auswirkungen vermindern [29].

\section{Stufe Trigger}

Im Entzündungsfall eignet sich die Akupunktur besonders gut zum Trig- gern antientzündlicher Mechanismen. Der anti-inflammatorische cholinergische Signalweg, von TraceY als „entzündlicher Reflex“ (inflammatory reflex) bezeichnet [2], spielt dabei eine wichtige Rolle. Ein erhöhter parasympathischer Output und das damit vom efferenten Vagus ausgeschüttete Acetylcholin hemmt über einen nikotinisch-cholinergen Rezeptor auf den Makrophagen deren Ausschüttung von proinflammatorischen Zytokinen (TNF- $\alpha$, IL-1 $\beta$ und IL-18) im Entzündungsherd [30]. Akupunktur hat aber auch über andere Signalwege antientzündliche und analgetische Wirkung. Vermutlich sind dafür sowohl zentral als auch peripher wirkende Prozesse verantwortlich [31], wie die Aktivierung der Hypothalamus-HypophysenNebennieren-Achse [32,33] sowie periphere Opioid-Mechanismen [34].

\section{Ernährungsstatus}

Viele Lebensumstände, z. B. basenarme Ernährung („fast und junk food“), aber auch Krankheiten, speziell chronische Erkrankungen, führen zu einer erhöhten Sympathikusaktivität mit vermehrter Entzündungsbereitschaft und latenter Azidose [35, 36, 37].

Für die ECM bedeutet dies, dass die im zirkadianen Rhythmus erfolgende Aufnahme, Speicherung und Abgabe von Stoffwechselprodukten gestört wird. Die vermehrt anfallenden sauren Moleküle binden an Kollagen und PG/GAGs der ECM und können schwerer wieder davon abgelöst und neutralisiert werden. Unter der latenten Azidose beginnt die ECM zu verschlacken. Wird die Bindungskapazität von ECM-Komponenten für die sauren Metaboliten und die Pufferfähigkeit im Extrazellulärraum überschritten, werden die Säuren ins Blut verschoben und belasten hier die Pufferkapazitäten. Ausserdem regen sie Makrophagen zur Bildung von proinflammatorischen Zytokinen an und induzieren in den Fibroblasten die Synthese inadäquater ECM-Komponenten wie z.B. zu stark säurebindendes Kollagen und schlecht vernetzte PG/GAGs. Damit verschlechtert sich die Funktion der ECM als Molekularsieb, was eine Vielfalt vegetativer, funktioneller und psychischer Symptome ohne eindeuti- ge Hinweise auf deren Ursachen zur Folge haben kann, wie z.B. chronisches Müdigkeitssyndrom, viszerale Hyperalgie, Kreuzschmerzen, Dysmenorrhoe und posttraumatisches Syndrom [35, 36, 37, 38].

\section{Stufe Reload}

Über geeignete Diätanpassungen kann die Regelfähigkeit der ECM wiederhergestellt werden. Die exogene Zufuhr von basischen Valenzen, z.B. direkt durch fixe Basen $\left(\mathrm{NaHCO}_{3}\right)$ und/oder durch Vollwertkost, erhöht die pH-Pufferkapazität [35]. Die Zufuhr von Antioxidantien bringt den Redoxstatus wieder ins Gleichgewicht, wobei zu beachten ist, dass verschieden starke Reduktionsmittel zugeführt werden, z.B. Vitamine und eine Vielzahl von sekundären Pflanzenstoffen wie sie in pflanzlichen Nahrungsmitteln vorliegen [39].

\section{Stufe Protect}

Die Erhaltung der Pufferkapazität bezüglich $\mathrm{pH}$ und Redoxstatus schützt die ECM vor Verschlackung durch sich anlagernde Säuren und durch Bildung von AGEs. Dadurch wird die Molekularsiebstruktur der ECM erhalten, die Bildung von proinflammatorischen Stimuli unterdrückt und der positive Feedbackmechanismus von ROI und AGEs unterbrochen. Beim Schutz der ECM-Komponenten vor Oxidation kommt auch der Verminderung des Eisenstatus eine entscheidende Bedeutung zu, da so die Entstehung von Fentonbedingungen gehemmt wird [40]. Auch die Versorgung mit essentiellen Fettsäuren spielt bei Entzündungsvorgängen eine Rolle, da mehrfachgesättigte Fettsäuren wie z.B. Omega-3-Fettsäuren anti-inflammatorisch wirken [41, 42, 43].

\section{Zusammenfassung in ein Therapieschema}

Die angeführten Beispiele Phytotherapie, Akupunktur und Ernährung zeigen, dass unterschiedliche komplementärmedizinische Behandlungsansätze im vorgestellten dreistufigen Matrix-basierten Interventionskonzept eingesetzt werden können und dass sie bei entzündlichen Erkrankungen auf verschiedenen Ebenen dieses Schemas wirken. 
In Tabelle 1 sollen nun in Form einer Arbeitsliste beispielhaft weitere häufig eingesetzte Behandlungsverfahren aufgeführt werden, jeweils mit einer Angabe, auf welcher Stufe des Matrix-basierten Therapieschemas sie angewendet werden können. Die Liste erhebt nicht den Anspruch auf Vollständigkeit und sie versteht sich nicht als Empfehlung einzelner Verfahren, sie zeigt jedoch auf, dass ganz unterschiedliche therapeutische Massnahmen als Bausteine eines individuellen Therapieprotokolles zur Behandlung, Prävention und Nachsorge von chronischen Entzündungsvorgängen herangezogen werden und auch kombiniert werden können. Damit wird einerseits eine methodenübergreifende integrative Sichtweise ermöglicht, zum anderen kann damit die Vollständigkeit des angewendeten Therapieprogramms geprüft werden in Bezug auf die für die ECM notwendige dreistufige Abfolge „Reload-Protect-Trigger“.

\section{Schlussfolgerungen}

Chronische Entzündungen sind bei vielen Erkrankungen ein eigener Teil der Pathogenese und richten lokal und systemisch oft mehr Schaden an als die eigentliche Krankheitsursache selbst. Die Behandlung ist schwierig, da eine Vielzahl von positiven Rückkoppelungsmechanismen die proinflammatorische Signalkaskade ständig in Bewegung hält.

Im komplexen Entzündungsprozess spielen nicht nur zelluläre Faktoren und die von ihnen produzierten Entzündungsmediatoren eine Rolle. Das chronisch-entzündliche Geschehen muss in einem erweiterten Kontext als Zusammenspiel der Zellen und des sie umgebenden Milieus als einer zusammenhängenden organisatorischen Einheit gesehen werden. In der ganzheitsmedizinischen Forschung wird dieses Konzept als das System der Grundregulation beschrieben.

Zur Beendigung von Entzündungsreaktionen, insbesondere von chronischen Entzündungen, ist deshalb nicht nur die Modulation von Zytokinen nötig, sondern die Wiederherstellung der Regelfähigkeit des Grundsystems ist

Tab. 1. Schema mit Beispielen antientzündlicher Behandlungsmethoden, welche in Prävention, Therapie und Nachsorge von chronisch-entzündlichen Erkrankungen eingesetzt werden. Die einzelnen Therapieansätze können nach ihrem Einfluss auf die drei Stufen "Reload-Protect-Trigger" gewichtet werden. Diese Arbeitsliste dient als Grundlage zur Erstellung eines individuellen, auf allen drei Stufen wirksamen Therapieplans, oder zur Überprüfung eines bestehenden Therapieprotokolls auf seine Wirksamkeit auf den verschiedenen Ebenen des Matrix-basierten Behandlungskonzeptes.

\begin{tabular}{|c|c|c|c|}
\hline & Reload & Protect & Trigger \\
\hline 1. Ernährungsstatus & $\mathbf{x}$ & $\mathbf{x x}$ & \\
\hline \multicolumn{4}{|l|}{ Übergewicht reduzieren } \\
\hline \multicolumn{4}{|l|}{ Minimierung des Eisenstatus } \\
\hline \multicolumn{4}{|l|}{ ausreichende Versorgung mit Omega-3-Fettsäuren } \\
\hline \multicolumn{4}{|l|}{$\begin{array}{l}\text { Diätmassnahmen zur Regulierung des Säure-Basen- } \\
\text { Haushalts }\end{array}$} \\
\hline 2. Verhaltens-, Lebensstilberatung & $\mathbf{x}$ & $\mathbf{x x}$ & \\
\hline \multicolumn{4}{|l|}{ Rauchstopp } \\
\hline \multicolumn{4}{|l|}{ Stressbewältigungsmethoden } \\
\hline \multicolumn{4}{|l|}{ ausreichend Bewegung, Sport } \\
\hline 3. Risikoprofil minimieren & $\mathbf{x}$ & $\mathbf{x x}$ & $\mathbf{x}$ \\
\hline \multicolumn{4}{|l|}{ Kontrolle von Diabetes, Hypertonie } \\
\hline \multicolumn{4}{|l|}{ Allergenkarenz } \\
\hline \multicolumn{4}{|l|}{ Störfaktoren und Störzonen mindern } \\
\hline \multicolumn{4}{|l|}{$\begin{array}{l}\text { Baubiologische Optimierung (z.B. Raumklima, } \\
\text { Wohngifte, Strahlungsfelder, Pilzbelastung) }\end{array}$} \\
\hline \multicolumn{4}{|l|}{ Baubiologisch optimiertes Raumklima } \\
\hline 4. Spezielle Supplementierung & $\mathbf{x x x}$ & $\mathbf{x}$ & \\
\hline \multicolumn{4}{|l|}{$\begin{array}{l}\text { Positiver Antioxidantien-Status: Vitamine (A, C, E), } \\
\text { Selen, Coenzym-Q10 }\end{array}$} \\
\hline \multicolumn{4}{|l|}{$\begin{array}{l}\text { Zufuhr von entzündungshemmenden Faktoren, } \\
\text { Zellschutz, Gemische sekundärer Pflanzenstoffe } \\
\text { (z.B. in Form von komplexen Phytotherapeutika } \\
\text { wie etwa solche aus der Tibetischen Medizin) }\end{array}$} \\
\hline 5. Ausleitung und Sanierung & $\mathbf{x}$ & $\mathbf{x x}$ & \\
\hline \multicolumn{4}{|l|}{$\begin{array}{l}\text { Sanierung von Antigenquellen (z.B. Herpes, } \\
\text { Chlamydien, Candida, Dentalplaque, Helicobacter) } \\
\text { vor allem via Milieuänderung }\end{array}$} \\
\hline \multicolumn{4}{|l|}{$\begin{array}{l}\text { Ausleiten von toxischen Substanzen, Förderung der } \\
\text { Ausscheidungsfunktionen (v.a. Leber, Nieren) }\end{array}$} \\
\hline \multicolumn{4}{|l|}{ Darmsanierung (z.B. leaky-gut Syndrome) } \\
\hline 6. Triggern antientzündlicher Mechanismen & & $\mathbf{x x}$ & $\mathbf{x x x}$ \\
\hline \multicolumn{4}{|l|}{ Meditation, Biofeedback, Hypnose } \\
\hline \multicolumn{4}{|l|}{$\begin{array}{l}\text { Akupunktur, Laserpunktur, Reflexpunktbehandlung, } \\
\text { Akupunktmassage }\end{array}$} \\
\hline \multicolumn{4}{|l|}{ Neuraltherapie } \\
\hline $\begin{array}{l}\text { gezielter, kurzfristiger Einsatz von Antiphlogistika } \\
\text { (nichtsteroidale Entzündungshemmer, Corticosteroide) }\end{array}$ & & & $\mathbf{x x x}$ \\
\hline
\end{tabular}

unerlässlich. Aus diesen Überlegungen lässt sich ein ganzheitsmedizinisches, antientzündliches Interventionsschema ableiten, dessen drei Stufen auf die Regeneration und Aufrechterhaltung der Funktionsfähigkeit der ECM ausgerichtet ist.

Anhand von Beispielen konnte ge- zeigt werden, dass sich diverse komplementärmedizinische Methoden für den Einsatz auf unterschiedlichen Stufen dieses Therapiekonzepts eignen. Die unterschiedlichen therapeutischen Ansätze lassen sich in ein praxistaugliches Schema einordnen, das die $\mathrm{Zu}$ sammenstellung eines individuellen 
Therapieplanes erlaubt, der in den drei Stufen „Reload-Protect-Trigger“ die Regelfähigkeit der ECM wieder herstellt. Die integrative Sichtweise erscheint auch insofern notwendig, als gerade im Bereich der chronischen Entzündungen eine Vielzahl von Massnahmen eingesetzt wird, eine übergeordnete Leitlinie jedoch oft fehlt.

Neben der möglichen praktischen Anwendung kann das vorgestellte Schema vielleicht dazu anregen, weitere integrative Behandlungskonzepte strukturiert darzustellen. Derartige Konzepte können bei der Hypothesenbildung der Grundlagenforschung bis hin zur Planung von Anwendungsbeobachtungen genutzt werden.

\section{Literatur}

1 Nathan C: Points of control in inflammation. Nature 2002;420:846-852.

2 Tracey KJ: The inflammatory reflex. Nature 2002;420:853-859.

3 Pajares JM, Gisbert JP: Helicobacter pylori: its discovery and relevance for medicine. Rev Esp Enferm Dig. 2006;98(10):770-785.

4 Benoist C, Mathis D: Mast cells in autoimmune disease. Nature 2002;420:875-878

5 Libby P: Inflammation in atherosclerosis. Nature 2002;420:868-874.

6 Schwabl H, Späni D: Das entzündliche Geschehen: Ganzheitsmedizinische Ansätze für die Therapie. Schweiz ZSchr Ganzheitsmedizin 2003;15:226-232

7 Theise N: Now you see it, now you don't. Nature 2005;435:1165.

8 Payne SL, Hendrix MJ, Kirschmann DA: Paradoxical roles for lysyl oxidases in cancer - A prospect. J Cell Biochem. 2007;doi: 10.1002/ jcb.21371 (Epub ahead of print).

9 Pischinger A: Das System der Grundregulation: Grundlagen einer ganzheitsbiologischen Medizin. 10. Auflage Haug-Verlag, Stuttgart 2004

10 Heine H: Grundregulation und extrazelluläre Matrix. 3. Auflage. Hippokrates-Verlag, Stuttgart 2006.

11 Heine $H$. Übergeordnete Regulationsprinzipien der extrazellulären Grundsubstanz (Matrix) für Prophylaxe und Regeneration. Schweiz Zschr GanzheitsMedizin 1989;2:77-83.

12 Navab R, Aingorn H, Fallavollita L, Sallon S, Mechoulam R, Ginsburg I, Vlodavsky I, Brodt P: Padma 28, a traditional Tibetan herbal preparation, blocks cellular responses to bFGF and IGF-I. Inflammopharmacology 2004;12:373-389.

13 Heine H: Grundregulation - eine Synthese medizinischen Denkens. Dt Ztschr $f$ Akup 2004;47:7-19.

14 Yan SF, Ramasamy R, Naka Y, Schmidt AM: Glycation, inflammation, and RAGE: a scaffold for the macrovascular complications of diabetes and beyond. Circ. Res. 2003;93(12) 1159-1169.

15 Ginsburg I, Sadovnic M, Oron M, Kohen R Novel chemiluminescence-inducing cocktails, part II: Measurement of the anti-oxidant capacity of vitamins, thiols, body fluids, alcoholic beverages and edible oils. Inflammopharmacology 2004;12:305-320.

16 Suter M, Richter Ch: Anti- and pro-oxidative properties of PADMA 28, a Tibetan herbal formula. Redox Rep 2000;5:17-22.

17 Vennos C, Schwabl H: Chronische Low-Leve Entzündungen: Pflanzliche Vielstoffgemische als integrative Erweiterung antioxidative Strategien. Schweiz Zschr GanzheitsMedizin 2006;18:333-338.

18 Sallon S, Beer G, Rosenfeld J, Anner H, Volcoff D, Ginsberg G, Paltiel O, Berlatzky Y: The efficacy of Padma 28, a herbal preparation, in the treatment of intermittent claudication: a controlled double-blind pilot study with objective assessment of chronic occlusive arteria disease patients. J Vasc Invest 1998:4:129-136.

19 Brunner-La Rocca HP, Schindler R, Schlump M, Saller R, Suter M: Effects of the Tibetan herbal preparation Padma 28 on blood lipids and lipid oxidisability in subjects with mild hypercholesterolaemia. VASA 2005;34:11-17.

20 Weseler A, Saller R, Reichling J: Comparative investigation of the antimicrobial activity of Padma 28 and selected European herbal drugs. Forsch Komplementarmed Klass $\mathrm{Na}$ turheilkd 2002;9:346-351.

21 Aslam MN, Fligiel H, Lateef H, Fisher GJ Ginsburg I, Varani J. Padma 28: A multi-component herbal preparation with retinoid-like dermal activity but without epidermal effects. J Invest Dermatol 2005;124: 524-9.

22 Ginsburg I, Sadovnik M, Sallon S, Milo-Goldzweig I, Mechoulam R, Breuer A, Gibbs D, Varani 'J, Roberts S, Cleator E, Singh N PADMA 28, a traditional Tibetan herbal preparation inhibits the respiratory burst in human neutrophils, the killing of epithelial cells by mixtures of oxidants and pro-inflammatory agonists and peroxidation of lipids. Inflammopharmacology 1999;7:47-62

23 Delhaye S, Paul S, Blakqori G, Minet M Weber F, Staeheli P, Michiels T: Neurons produce type I interferon during viral encephalitis. Proc Natl Acad Sci USA. 2006;103(20): 7835-7840.

24 Rempel JD, Quina LA, Blakely-Gonzales PK Buchmeier MJ, Gruol DL: Viral induction of central nervous system innate immune responses. J. Virol. 2005;79(7):4369-481.

25 Dunn AJ, Wang J: Cytokine effects on CNS biogenic amines. Neuroimmunomodulation. 1995;2(6):319-328.

26 Kim SK, Lee G, Shin M, Han JB, Moon HJ, Park JH, Kim KJ, Ha J, Park DS, Min BI: The association of serum leptin with the reduction of food intake and body weight during electroacupuncture in rats. Pharmacol Biochem Behav 2006;83:145-149.

27 Cabyoglu MT, Ergene N, Tan U: The treatment of obesity by acupuncture. Int J Neurosci 2006;116:165-175.

28 White AR, Rampes H, Campbell JL: Acupuncture and related interventions for smok ing cessation. Cochrane Database Syst Rev 2006 Jan 25:CD000009.

29 Iwa M, Nakade Y, Pappas TN, Takahashi T: Electroacupuncture Elicits Dual Effects: Stimulation of Delayed Gastric Emptying and Inhibition of Accelerated Colonic Transit Induced by Restraint Stress in Rats. Dig Dis Sci. 2006 Epub ahead of print; DOI: 10.1007/s10620006-9083-7.

30 Pavlov VA, Wang H, Czura CJ, Friedman SG, Tracey KJ: The cholinergic anti-inflammatory pathway: a missing link in neuroimmunomodulation. Mol Med 2003;9:125-134.

31 Ceccherelli F, Gagliardi G, Ruzzante L, Giron $\mathrm{G}$ : Acupuncture modulation of capsaicin- induced inflammation: effect of intraperitoneal and local administration of naloxone in rats. A blinded controlled study. J Altern Complement Med 2002;8:341-149.

32 Cho ZH, Hwang SC, Wong EK, Son YD, Kang CK, Park TS, Bai SJ, Kim YB, Lee YB, Sung KK, Lee BH, Shepp LA, Min KT: Neural substrates, experimental evidences and functional hypothesis of acupuncture mechanisms. Acta Neurol Scand 2006;113:370-377.

33 Zhang $R X$, Lao L, Wang $X$, Fan A, Wang L, Ren K, Berman BM: Electroacupuncture attenuates inflammation in a rat model. J Altern Complement Med 2005;11:135-142.

34 Kim HW, Roh DH, Yoon SY, Kang SY, Kwon YB, Han HJ, Lee HJ, Choi SM, Ryu YH, Beitz AJ, Lee JH: The anti-inflammatory effects of low- and high-frequency electroacupuncture are mediated by peripheral opioids in a mouse air pouch inflammation model. J Altern Complement Med 2006;12:39-44.

35 Vormann J, Goedecke T: Acid-Base Homeostasis: Latent Acidosis as a Cause of Chronic Diseases. Schweiz Zschr GanzheitsMedizin 2006:18:255-266.

36 Heine $\mathrm{H}$ : Die extrazelluläre Matrix als Attraktor für Verschlackunsphänomene. in Marktl W, Reiter B, Ekmekcioglu C (eds). Säuren Basen - Schlacken; Pro und Contra - eine wissenschaftliche Diskussion. Springer Wien; Auflage 1; 2007;51-56

37 Kellum JA, Song M, Li J: Science review: Extracellular acidosis and the immune response: clinical and physiologic implications. Critical Care 2004:8:331-336.

38 Vormann J, Worlitschek M, Goedecke T, Silver B: Supplementation with alkaline minerals reduces symptoms in patients with chronic low back pain. J Trace Elem Med Biol. 2001;15:179-183.

39 Vennos C, Schwabl H: Chronische Low-Level Entzündungen: Pflanzliche Vielstoffgemische als integrative Erweiterung antioxidativer Strategien. Schweiz Zschr GanzheitsMedizin 2006;18:333-338.

40 Kieffer F: Eisenüberladung und oxidativer Stress. Forsch Komplementärmed 1995:2; 259-267.

41 Hudert CA, Weylandt KH, Lu Y, Wang J, Hong S, Dignass A, Serhan CN, Kang JX: Transgenic mice rich in endogenous omega-3 fatty acids are protected from colitis. Proc. Natl. Acad. Sci. USA. 2006;103:11276-11281.

42 Arita M, Bianchini F, Aliberti J, Sher A, Chiang N, Hong S, Yang R, Petasis NA, Serhan CN: Stereochemical assignment, antiinflammatory properties, and receptor for the omega-3 lipid mediator resolvin E1. J. Exp. Med. 2005 Mar 7;201(5):713-22.

43 Saller R, Römer-Lüthi Chr, Müller M, Brignoli $R$, Noll G, Meier R: Docosahexaensäure (DHA) und langkettige Omega-3-Fettsäuren: Klinische Bedeutung bei entzündlichen und anderen Erkrankungen. Schweiz Zschr GanzheitsMedizin 2006:18:321-327.

\section{Korrespondenzadresse:}

Dr. techn. Herbert Schwabl

Padma AG

Wiesenstrasse 5

$\mathrm{CH}-8603$ Schwerzenbach

h.schwabl@padma.ch 